Research Paper

\title{
The Prognostic and Predictive Value of Carbohydrate Antigen 19-9 in Metastatic Colorectal Cancer Patients with First Line Bevacizumab Containing Chemotherapy
}

\author{
Chang Jiang1,2,3*, Shousheng Liu1,2,3*, Wenzhuo He ${ }^{1,2,3}$, Bei Zhang ${ }^{1,2,3 凶}$, Liangping Xia ${ }^{1,2,3 凶}$ \\ 1. VIP Region, Sun Yat-Sen University Cancer Center, Guangzhou, Guangdong, 510060, R.P. China; \\ 2. State Key Laboratory of Oncology in South China, Sun Yat-Sen University Cancer Center, Guangzhou, Guangdong, 510060, R.P. China; \\ 3. Collaborative Innovation Center for Cancer Medicine, Guangzhou, Guangdong, 510060, R.P. China. \\ * contributed equally to this paper \\ $\triangle$ Corresponding authors: Liangping Xia, E-mail: xialp@sysucc.org.cn, Fax: +86 20-87343392 Tel: +86 20-87343107 Mobile: +86-13926410608, Co-corresponding \\ author: Bei Zhang.
}

(c) Ivyspring International Publisher. This is an open access article distributed under the terms of the Creative Commons Attribution (CC BY-NC) license (https://creativecommons.org/licenses/by-nc/4.0/). See http://ivyspring.com/terms for full terms and conditions.

Received: 2016.11.13; Accepted: 2017.02.03; Published: 2017.05.12

\begin{abstract}
Objective: We had previously demonstrated that the carbohydrate antigen 19-9 (CA19-9), lactate dehydrogenase (LDH), neutrophil lymphocyte ratio (NLR) are prognostic factors for patients with metastatic colorectal cancer ( $m C R C)$. In this study, we try to analysis the association of these blood-based biomarkers with bevacizumab efficacy in the first line setting.

Methods: A total of 284 eligible consecutive mCRC patients who received first-line chemotherapy with or without bevacizumab were studied from 2007 to 2014 at Sun Yat-Sen University Cancer Center. The endpoints were overall survival (OS), progression free survival (PFS).

Results: Among all the patients, the initial elevated CA19-9, high LDH, and NLR $>2.47$ were confirmed as independent unfavorable prognostic factors. The CA19-9 and LDH levels were significantly associated with PFS. In the high CA19-9 subgroup, patients had favorable OS from bevacizumab administration in the first line therapy ( 32.1 vs. 20.1 months, $P=0.03$ ), but without PFS benefit. In terms of different levels of LDH, and NLR, there were no survival benefit from bevacizumab treatment.

Conclusions: Our results suggest that the initial CA19-9, LDH, and NLR levels could be independent prognostic biomarkers in $\mathrm{mCRC}$ patients. And among all these factors, the initial high CA19-9 level could be a predictor for bevacizumab effect.
\end{abstract}

Key words: carbohydrate antigen 19-9, bevacizumab, predictive marker, overall survival, metastatic colorectal cancer.

\section{Introduction}

The colorectal cancer is one of the most frequently diagnosed and deadly cancer globally [1]. Nearly $40-50 \%$ colorectal cancer patients were initially diagnosed with metastatic lesion(s) [2]. Due to distinct outcomes among metastatic colorectal cancer (mCRC) patients, various efforts had been attempted for systematic management. In the clinical practice, the measurement of tumor markers such as carcinoembryonic antigen (CEA), carbohydrate antigen 19-9 (CA19-9) are commonly used during the management of colorectal cancer patients [3]. Besides, other readily available indexes associated with the cancer-related metabolism and inflammation processes could be surrogate markers including lactate dehydrogenase (LDH) [4, 5], neutrophil lymphocyte ratio (NLR) [6, 7] for evaluating the 
prognosis. In our previous study, we have also found that increased CEA [8], high CA19-9 [9], and upraised NLR [10] are adverse prognostic indicators.

As an encouraging targeting tumor microvascular monoclonal antibody bevacizumab had been widely administrated in mCRC [11-13]. However, as the angiogenesis process in $\mathrm{MCRC}$ is not completely discerned, the optimal predictive factor for bevacizumab has not been discovered yet [14, 15]. Our earlier study suggested that the LDH in peripheral blood would serve as a potential predictor of bevacizumab therapy [16]. Nonetheless, the several isoforms could hardly make LDH a satisfactory biomarker candidate.

The objective of our present study was to assess the association of baseline serum CEA, CA 19-9, LDH, and NLR with outcomes of mCRC patients and bevacizumab effect.

\section{Materials and Methods}

\section{Patients}

We retrospectively analyzed the intact data of 284 patients who were pathological diagnosed with mCRC and with clinical and/or pathological confirmations of metastasis at Sun Yat-Sen University Cancer Center from January 2005 to March 2014 and finished the integrated course of first-line treatment in this center. Patients in chemotherapy plus bevacizumab group received more than 4 cycles bevacizumab (at a dose of $5 \mathrm{mg} / \mathrm{kg}$ on day 1) [17] was administered as a combination with the standard oxaliplatin-based or irinotecan-based first-line chemotherapy. None of the patients had previously or subsequently received other target therapies. Patients who lack of a pathological diagnosis or complete medical history, loss to follow-up, suffering from two or more kinds of tumors asynchronously or synchronously were excluded.

\section{Laboratory measurement, calculation and grouping}

Neutrophil and lymphocyte counts were contained in complete blood counts test by the Sysmex XE-5000 ${ }^{\mathrm{TM}}$ Automated Hematology System (Shanghai, China). NLR was calculated as neutrophil count (number of neutrophils $\times 10^{9} / \mathrm{L}$ ) divided by lymphocyte count (number of lymphocytes $\times 10^{9} / \mathrm{L}$ ), using the median value 2.47 for cutoff. While LDH was contained in biochemical test by the Hitachi Automatic Analyzer 7600-020 (Tokyo, Japan). Roche Elecsys 2010 Chemistry Analyzer (Basel, Switzerland) was used to test CEA and CA19-9. The upper normal values were used as cutoff: CEA $5 \mathrm{ng} / \mathrm{ml}$; CA 19-9 35 $\mathrm{ng} / \mathrm{ml}$; LDH $245 \mathrm{U} / \mathrm{ml}$.

\section{Follow up and statistical analysis}

The latest follow-up was performed on June 30ed, 2016 through telephone interview or medical records review. Overall survival (OS) was measured from date of diagnosis with mCRC to the date of death. Progression free survival (PFS) was measured from the initiation of first line therapy to the progression. Both OS and PFS were estimated by the Kaplan-Meier method and survival differences were analyzed by the log-rank test. The distributions of the baseline characteristics of the patients were assessed by the $X^{2}$ test. The $P$ value less than 0.05 was considered with statistically significant.

\section{Human Rights}

The protocol of our study was approved by the independent ethics committee of the Sun Yat-Sen University Cancer Center and complied with the International Ethical Guidelines for Biomedical Research Involving Human Subjects, Good Clinical Practice guidelines, the Declaration of Helsinki, and local laws. Written informed consent was gathered from each patient.

\section{Results}

\section{Patient characteristics}

A total of 284 patients were enrolled in this retrospective study, including 59 patients received chemotherapy containing bevacizumab $(B+C$ group) and 225 patients received chemotherapy alone (C group). The patient characteristics between the two groups are shown in Table 1. All of the factors were balanced in statistics. As a main clinic parameter, the bevacizumab treatment was separately statistically compared, making it difficult to judge whether these two groups were comparable according to initial levels of serum indexes (Table 2). The bevacizumab administrations were balanced within different subgroup of several serum indexes.

\section{Relation between initial levels of serum indexes and survival}

Initial serum levels of CEA, CA 19-9, LDH, and NLR are shown in Table 3. The median PFS and OS for patients with low CEA was 12.0 and 33.7 months compared to 9.1 and 25.5 months for patients with high CEA $(P=0.002$ and $P=0.008)$ (Figure 1A, B). The median PFS and OS for patients with low CA 19-9 was 11.4 and 35.7 compared to 7.9 and 22.3 months for patients with high CA $19-9(P<0.001$ and $P<0.001)$ (Figure 2A, B). The median PFS and OS for patients with low LDH was 10.8 and 33.0 compared to 8.2 and 19.5 months for patients with high LDH $(P<0.001$ and $P<0.001$ ) (Figure 3A, B). The median PFS and OS for 
patients with low NLR was 10.8 and 32.6 compared to 8.2 and 22.4 months for patients with high NLR $(P=$ 0.002 and $P=0.001$ ) (Figure $4 \mathrm{~A}, \mathrm{~B}$ ).

Table 1. Patient characteristics

\begin{tabular}{ll}
\hline Characteristics & $n(\%$, total n=284) \\
\hline Males & $187(65.8)$ \\
Age (years) & \\
median, range & $55(19-87)$ \\
$\leq 60$ & $191(67.3)$ \\
$>60$ & $93(32.7)$ \\
Primary Tumor & \\
Right colon & $87(30.6)$ \\
Left colon & $95(33.5)$ \\
Rectum & $102(35.9)$ \\
Pathological type & \\
Well differentiation & $3(1.1)$ \\
Moderately differentiation & $177(63.3)$ \\
Poorly differentiation & $64(22.5)$ \\
Mucinous adenocarcinoma & $35(12.3)$ \\
Unknown a & $5(1.8)$ \\
Pathologic tumor classification & \\
T1 & $4(1.4)$ \\
T2 & $9(3.2)$ \\
T3 & $50(17.6)$ \\
T4 & $219(77.1)$ \\
Unknown b & $2(0.7)$ \\
Lymphatic invasion & $201(70.8)$ \\
Vascular invasion & $26(9.2)$ \\
Perineural invasion & $8(2.8)$ \\
First line bevacizumab therapy & \\
With Bevacizumab & $59(20.8)$ \\
Without Bevacizumab & $225(79.2)$ \\
First line chemotherapy regimen & \\
Oxaliplatin-based & $213(75.0)$ \\
Irinotecan-based & $53(18.7)$ \\
Fluorouracil alone & $5(1.8)$ \\
Oxaliplatin plus irinotecan & $13(4.5)$ \\
\hline a This part of patients was pathological diagnosed with colorectal adenocarcinoma \\
without the differentiation degree by the biopsy specimen or pathology \\
consultation of specimen from other hospitals; \\
b There were no surgical primary tumor specimen of these patients. \\
\end{tabular}

Table 2. Statistically comparison of bevacizumab treatment according to initial levels of serum indexes

\begin{tabular}{|c|c|c|c|}
\hline & $\begin{array}{l}\text { With bevacizumab } \\
n(\%)\end{array}$ & $\begin{array}{l}\text { Without bevacizumab } \\
n(\%)\end{array}$ & $P$ \\
\hline CEA (ng/ml) & & & 0.87 \\
\hline$\leq 5$ & 15 (19.5) & $62(80.5)$ & \\
\hline$>5$ & $44(21.3)$ & 163 (78.7) & \\
\hline $\begin{array}{l}\text { CA19-9 } \\
\text { (ng/ml) }\end{array}$ & & & 0.77 \\
\hline$\leq 35$ & $30(21.7)$ & 108 (78.3) & \\
\hline$>35$ & $29(19.9)$ & 117 (80.1) & \\
\hline LDH (U/ml) & & & 0.94 \\
\hline$\leq 245$ & $43(20.7)$ & 165 (79.3) & \\
\hline$>245$ & 16 (21.1) & $60(78.9)$ & \\
\hline NLR & & & 0.55 \\
\hline$\leq 2.47$ & $29(20.7)$ & 111 (79.3) & \\
\hline$>2.47$ & $30(20.8)$ & 114 (79.2) & \\
\hline
\end{tabular}

Table 3. Progression free survival and overall survival according to initial levels of serum indexes

\begin{tabular}{|c|c|c|c|c|c|}
\hline & $\mathrm{n}(\%)$ & $\begin{array}{l}\text { median PFS } \\
\text { (months, 95\% CI) }\end{array}$ & $P$ & $\begin{array}{l}\text { median OS } \\
\text { (months, } \\
95 \% C l \text { ) }\end{array}$ & $P$ \\
\hline CEA (ng/ml) & & & 0.002 & & 0.008 \\
\hline$\leq 5$ & $77(27.1)$ & $12.0(9.6-14.4)$ & & 33.7 (26.9-40.5) & \\
\hline$>5$ & 207 (72.9) & $9.1(8.0-10.2)$ & & $25.5(21.6-29.4)$ & \\
\hline CA19-9 (ng/ml) & & & $<0.001$ & & $<0.001$ \\
\hline$\leq 35$ & $102(35.9)$ & 11.4 (9.6-13.3) & & 35.7 (30.5-40.9) & \\
\hline$>35$ & 118 (41.1) & $7.9(6.6-9.3)$ & & $22.3(18.0-26.5)$ & \\
\hline LDH (U/ml) & & & $<0.001$ & & $<0.001$ \\
\hline$\leq 245$ & 208 (73.3) & $33.0(28.9-37.2)$ & & $10.8(9.3-12.3)$ & \\
\hline$>245$ & $76(26.7)$ & $19.5(16.0-23.0)$ & & $8.2(6.7-9.8)$ & \\
\hline NLR & & & 0.002 & & 0.001 \\
\hline$\leq 2.47$ & $140(49.3)$ & $10.8(9.3-12.3)$ & & 32.6 (29.7-35.5) & \\
\hline$>2.47$ & $144(50.7)$ & $8.2(6.9-9.6)$ & & $22.4(18.6-26.3)$ & \\
\hline
\end{tabular}

All the prognostic serum factors were assessed together in multivariate Cox proportional hazards model. The multivariate Cox proportional hazards model revealed that the CA19-9 $(H R=1.45, P=0.004)$, $\mathrm{LDH}(H R=1.37, P=0.04)$ were significantly associated with PFS, and the CA19-9 $(H R=1.60, P=$ $0.01), \mathrm{LDH}(H R=1.84, P<0.001), \mathrm{NLR}(H R=1.38, P=$ 0.04 ) were significantly associated with OS (Table 4).

Table 4. Hazard ratios from multivariate Cox proportional hazard model for progression free survival and overall survival

\begin{tabular}{lllll}
\hline Index & PFS & \multicolumn{3}{l}{ OS } \\
\cline { 2 - 5 } & HR $(95 \% \mathrm{Cl})$ & $P$ & $\mathrm{HR}(95 \% \mathrm{Cl})$ & $P$ \\
\hline CEA & $1.23(0.91-1.67)$ & 0.12 & $1.05(0.75-1.45)$ & 0.8 \\
CA19-9 & $1.45(1.12-1.88)$ & 0.004 & $1.60(1.21-2.13)$ & 0.001 \\
LDH & $1.37(1.02-1.84)$ & 0.04 & $1.84(1.35-2.50)$ & $<0.001$ \\
NLR & $1.27(0.96-1.68)$ & 0.1 & $1.38(1.01-1.87)$ & 0.04 \\
\hline
\end{tabular}

CEA carcinoembryonic antigen; CA 19-9 carbohydrate antigen 19-9, LDH lactate dehydrogenase, NLR neutrophil lymphocyte ratio, PFS progression free, OS overall survival, HR hazard ratio, $\mathrm{CI}$ confidence interval

\section{Relation between initial levels of serum indexes and bevacizumab efficacy}

On the basis of whether the first line therapy containing bevacizumab or not, the patients could be further divided into bevacizumab plus chemotherapy $(\mathrm{B}+\mathrm{C}$ group) and chemotherapy group ( $\mathrm{C}$ group). In the high CA19-9 subgroup, patients had favorable OS from bevacizumab treatment in the first line (32.1 vs. 20.1 months, $P=0.03$, Figure 5A), though without progression free survival benefit (10.6 vs. 7.2 months, $P=0.07$, Figure 5B). When it comes to low CA19-9 subgroup, CEA, LDH, and NLR subgroups, there were no statistically survival difference between $B+C$ group and $\mathrm{C}$ group (Table 5 ). 
A

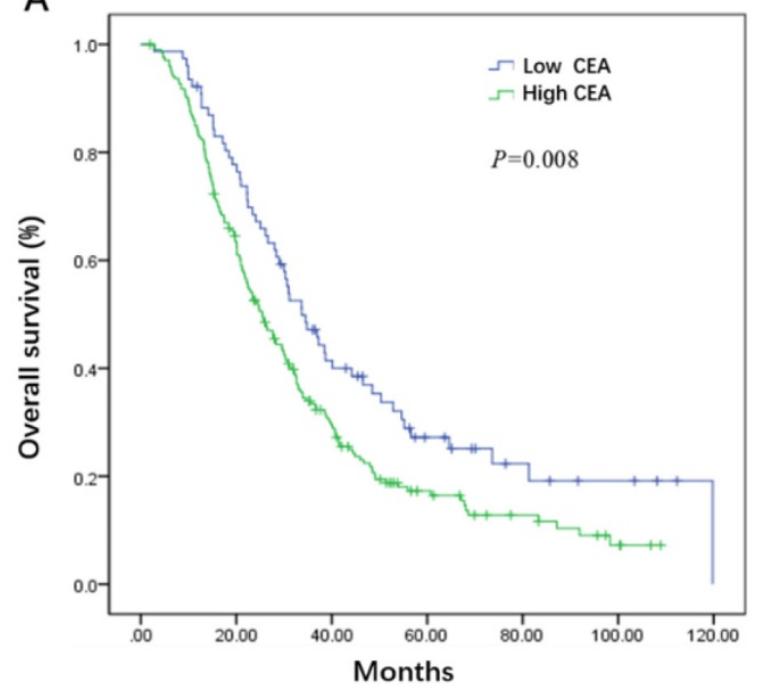

B

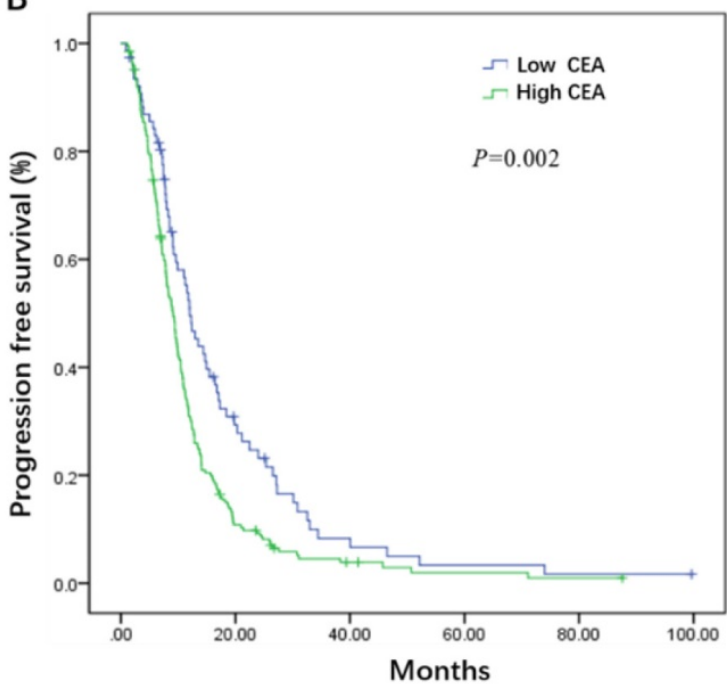

Figure 1. Kaplan-Meier estimates of overall survival (OS) (A) and progression-free survival (PFS) (B) according to the initial levels of carcinoembryonic antigen (CEA)
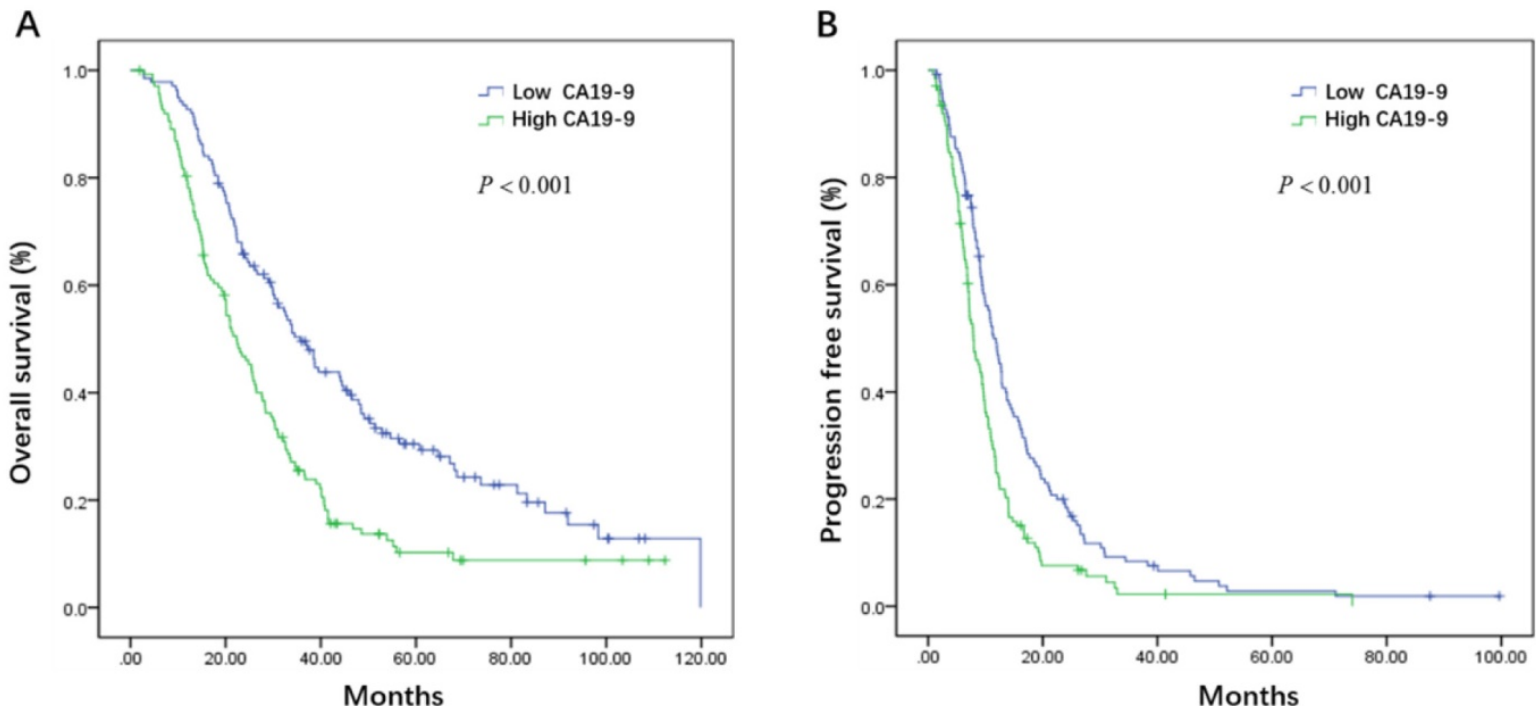

Figure 2. Kaplan-Meier estimates of overall survival (OS) (A) and progression-free survival (PFS) (B) according to the initial levels of carbohydrate antigen $19-9$ (CA 19-9)

A

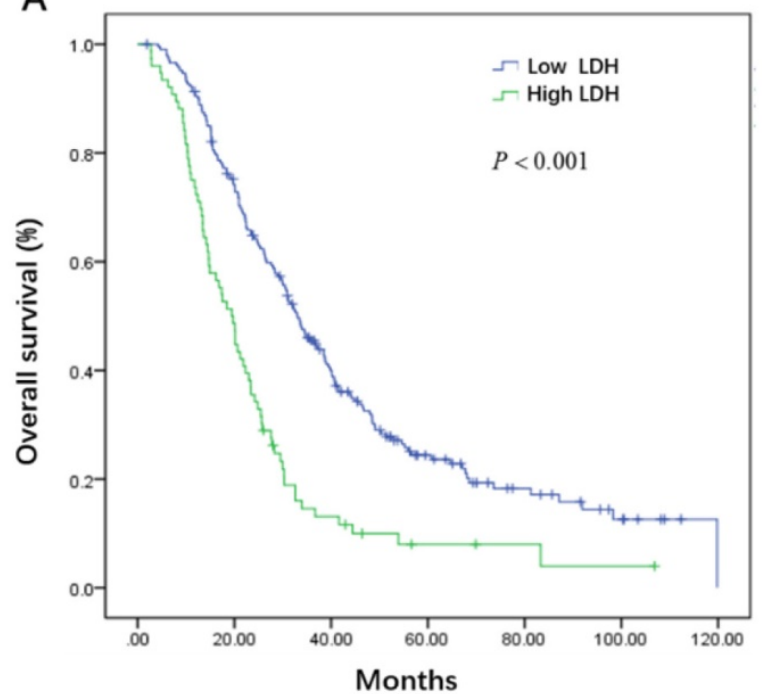

B

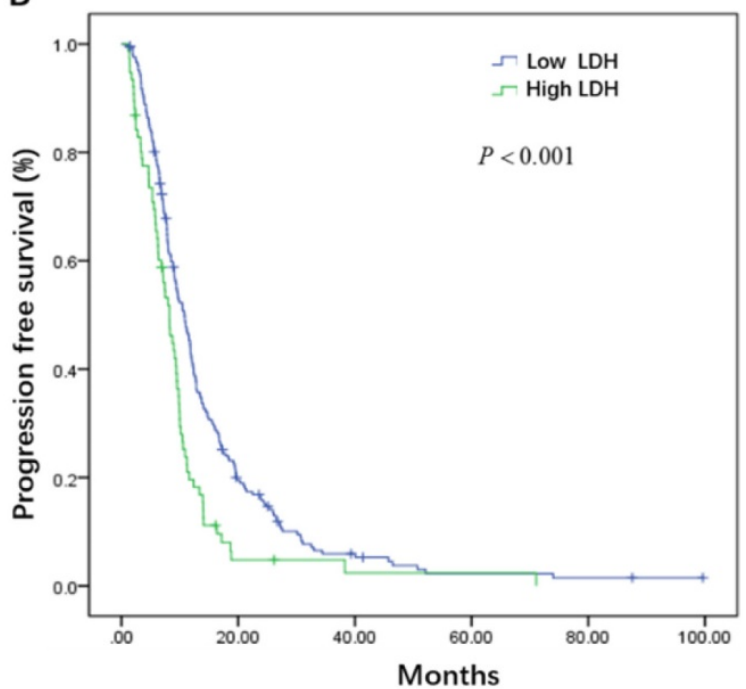

Figure 3. Kaplan-Meier estimates of overall survival (OS) (A) and progression-free survival (PFS) (B) according to the initial levels of lactate dehydrogenase (LDH) 
A

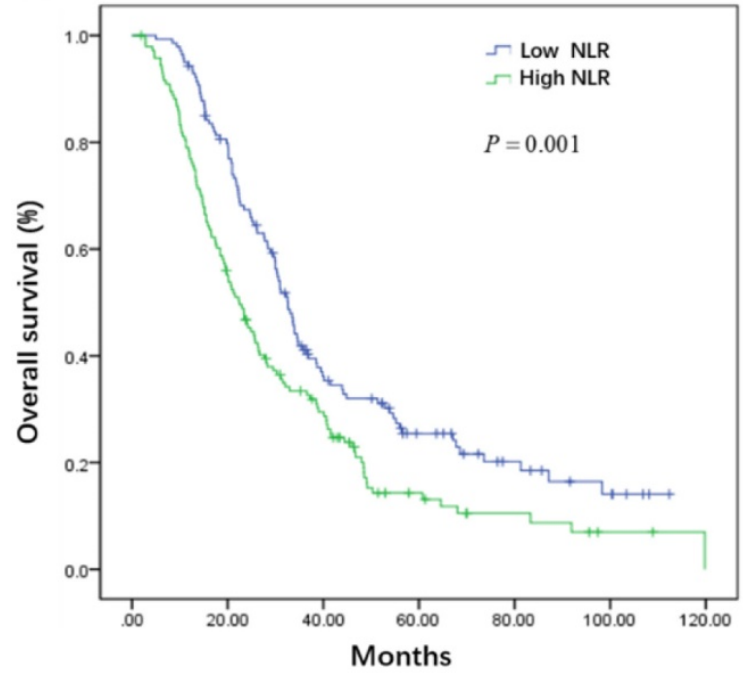

B

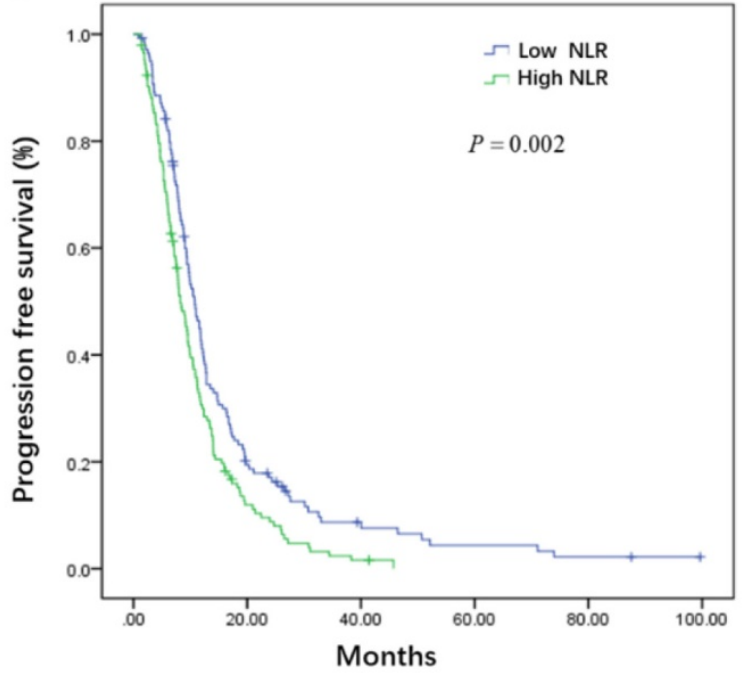

Figure 4. Kaplan-Meier estimates of overall survival (OS) (A) and progression-free survival (PFS) (B) according to the initial levels of neutrophil lymphocyte ratio (NLR)

A

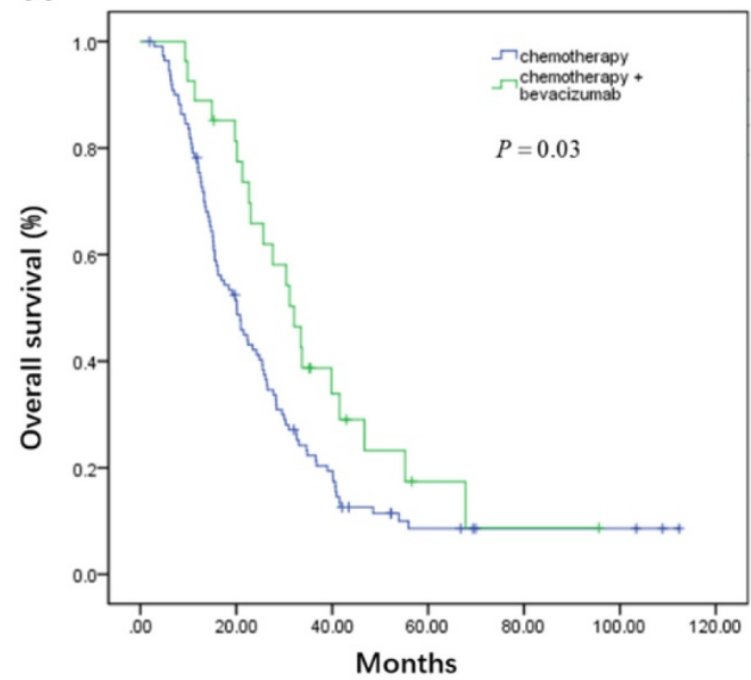

B

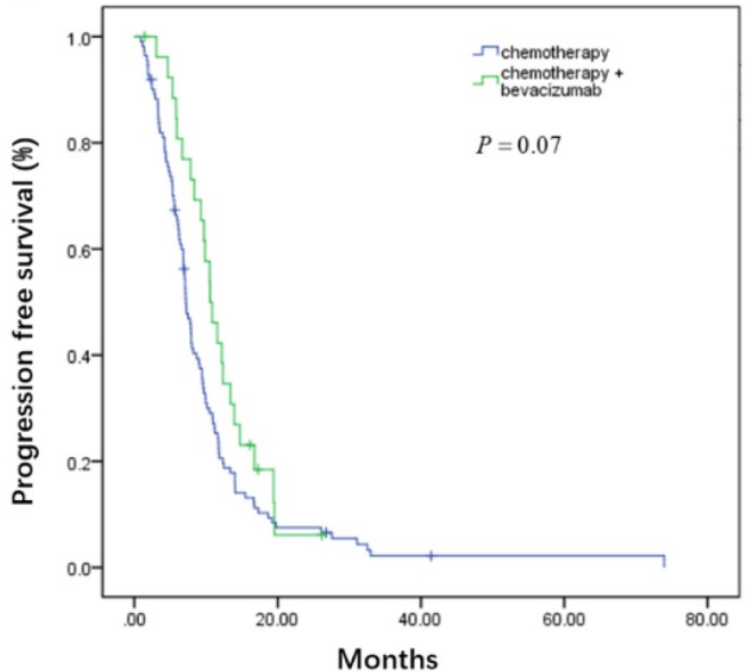

Figure 5. Kaplan-Meier estimates of overall survival (OS) (A) and progression-free survival (PFS) (B) according to whether adding bevacizumab in the first line setting in the high carbohydrate antigen 19-9 (CA19-9) subgroup

Table 5. Association of bevacizumab efficacy and initial levels of serum indexes

\begin{tabular}{|c|c|c|c|c|c|c|}
\hline & \multicolumn{2}{|c|}{ median PFS (months, 95\% CI) } & \multirow[t]{2}{*}{$P$} & \multicolumn{2}{|c|}{ median OS (months, 95\% CI) } & \multirow[t]{2}{*}{$P$} \\
\hline & $\begin{array}{l}\mathrm{B}+\mathrm{C} \text { group } \\
\mathrm{n}=59\end{array}$ & $\begin{array}{l}C \text { group } \\
n=225\end{array}$ & & $\begin{array}{l}\mathrm{B}+\mathrm{C} \text { group } \\
\mathrm{n}=59\end{array}$ & $\begin{array}{l}\text { C group } \\
n=225\end{array}$ & \\
\hline \multicolumn{7}{|c|}{ CEA (ng/ml) } \\
\hline$\leq 5$ & $16.4(8.8-24.0)$ & $12.0(9.0-15.0)$ & 0.4 & $46.5(25.2-67.9)$ & $33.7(26.4-41.0)$ & 0.9 \\
\hline$>5$ & 10.6(10.0-11.1) & $8.2(7.0-9.4)$ & 0.3 & $29.6(22.6-36.6)$ & $24.6(20.7-28.5)$ & 0.2 \\
\hline \multicolumn{7}{|c|}{ CA19-9 (ng/ml) } \\
\hline$\leq 35$ & $12.8(7.8-17.8)$ & $11.2(8.9-13.5)$ & 0.80 & $36.9(31.7-42.2)$ & $30.3(8.6-52.0)$ & 0.70 \\
\hline$>35$ & $10.6(8.4-12.7)$ & $7.2(6.4-8.0)$ & 0.07 & $32.1(24.8-39.4)$ & $20.1(15.8-24.4)$ & 0.04 \\
\hline \multicolumn{7}{|c|}{$\mathrm{LDH}(\mathrm{U} / \mathrm{ml})$} \\
\hline$\leq 245$ & $12.2(10.1-14.3)$ & $9.4(7.3-11.5)$ & 0.4 & $39.9(24.0-55.8)$ & $32.3(27.8-36.9)$ & 0.4 \\
\hline$>245$ & $8.3(6.0-10.7)$ & $8.0(6.0-9.9)$ & 0.3 & 20.1(17.1-23.1) & $17.5(12.6-22.4)$ & 0.7 \\
\hline \multicolumn{7}{|l|}{ NLR } \\
\hline$\leq 2.47$ & $12.8(9.0-16.6)$ & $9.8(8.3-11.3)$ & 0.4 & $34.0(22.3-45.6)$ & $32.3(29.2-35.4)$ & 0.8 \\
\hline$>2.47$ & $10.4(8.8-12.0)$ & $7.8(6.8-9.0)$ & 0.4 & $23.4(14.8-32.0)$ & $21.5(16.5-26.6)$ & 0.2 \\
\hline
\end{tabular}

CEA carcinoembryonic antigen, CA 19-9 carbohydrate antigen 19-9, LDH lactate dehydrogenase, NLR neutrophil lymphocyte ratio, PFS progression free, OS overall survival, $\mathrm{CI}$ confidence interval, $\mathrm{C}$ chemotherapy, $\mathrm{B}+\mathrm{C}$ bevacizumab plus chemotherapy 


\section{Discussion}

Bevacizumab, the classical anti-angiogenesis drug, had been widely applied in various cancers [18]. Meanwhile, hunting for suitable predictor of bevacizumab efficacy continues. Several surrogates including circulating vascular endothelial growth factor A (VEGF-A) isoforms, vascular endothelial growth factor receptor-1,2 (VEGFR-1, and VEGFR-2) had been explored but not sufficiently validated for routine clinical use $[15,19]$. CEA, CA19-9, LDH, NLR are readily available serum biomarkers in diagnosing and monitoring the mCRC patients. Therefore, we paid attention to the correlation between these indexes and bevacizumab efficacy.

As an intercellular adhesion molecular of immunoglobulin superfamily, CEA promotes the gathering of tumor cells [20]. It is reported CEA had a specificity of $90 \%$ and a sensitivity of $40 \%$ to $75 \%$ in colorectal cancer [3]. Albeit CEA is a widely accepted prognostic biomarker in colorectal cancer [21, 22], it is still dubious in several other studies [23-25] in which CEA showed no statistically significant prognostic value. In our study, we found a significantly shorter PFS and OS in patients with high initial CEA levels. However, it did not prove to be an independent biomarker in the multivariate Cox proportional hazards model. Moreover, there was no association between baseline CEA levels and the efficacy of bevacizumab based therapy.

Though CA19-9 frequent elevation caused by pancreatitis, obstructive jaundice and pancreatic cancer [26], it's still a critical additional marker to monitor colorectal cancer [27]. Almost 35\% to $40 \%$ of colorectal cancer patients had increased CA19-9 level [28]. CA19-9 had been found promoting adhesion of cancer cells to endothelial [29]. Similarly, we observed high initial CA19-9 level associated with a significantly poor PFS and OS, and the multivariate Cox proportional hazards model confirmed that CA 19-9 was independent prognostic marker. On the other hand, several studies [30-32] have reported that patients received bevacizumab plus chemotherapy have promising OS compared with chemotherapy alone in the high pre-treatment CA19-9 level group. While there was no similar difference in normal CA19-9 group. Fiala $\mathrm{O}$ et al. [33] had observed the analogous results among patients with bevacizumab treatment. Likewise, our data indicated the potential predictive role of CA19-9 in bevacizumab therapy.

$\mathrm{LDH}$, the main catalyst of aerobic glycolysis, reflects the metabolic status of tumor cells. High serum LDH level had been demonstrated to be a unfavorable prognostic factor in several tumors [34-36]. Our previous study has also explored the associated of the serum LDH, the outcome and the bevacizumab efficacy [16]. In our present study, the lack of correlation between serum LDH and bevacizumab efficacy, may enlighten the further exploration of local LDH in tumor microenvironment.

Increased NLR was associated with poor tumor outcome had been observed before [10,37], implying the interaction of inflammatory and immune response play a role in tumor progression. Our data here is consistent with this conclusion.

The main limitations of this study are its retrospective design and relatively small number of patients with bevacizumab therapy.

In conclusion, the initial CA19-9, LDH level were independently associated with PFS, while the baseline CA19-9, LDH, and NLR level independently associated with OS in first line treatment. The pre-treatment CA19-9 level could potentially serve as a predictor of bevacizumab. Prospective studies on the predictive role of serum markers should be conducted to certify the results.

\section{Acknowledgements}

This study was supported by grants from the Natural Science Foundation of Guangdong, China (2015A030313010), Science and Technology Program of Guangzhou, China (1563000305) and National Natural Science Foundation of China (81272641and 81572409).

\section{Competing Interests}

The authors have declared that no competing interest exists.

\section{References}

1. Torre LA, Bray F, Siegel RL, Ferlay J, Lortet-Tieulent J, Jemal A. Global cancer statistics, 2012. CA: a cancer journal for clinicians. 2015; 65: 87-108.

2. Grothey A, Van Cutsem E, Sobrero A, Siena S, Falcone A, Ychou M, et al. Regorafenib monotherapy for previously treated metastatic colorectal cancer (CORRECT): an international, multicentre, randomised, placebo-controlled, phase 3 trial. Lancet (London, England). 2013; 381: 303-12.

3. Stiksma J, Grootendorst DC, van der Linden PW. CA 19-9 as a marker in addition to CEA to monitor colorectal cancer. Clinical colorectal cancer. 2014; 13: $239-44$.

4. Armstrong AJ, George DJ, Halabi S. Serum lactate dehydrogenase predicts for overall survival benefit in patients with metastatic renal cell carcinoma treated with inhibition of mammalian target of rapamycin. Journal of clinical oncology : official journal of the American Society of Clinical Oncology. 2012; 30: 3402-7.

5. Kostakis ID, Vaiopoulos AG, Philippou A, Papavassiliou AG, Koutsilieris M, Kouraklis G. Preoperative serum lactate dehydrogenase levels in colorectal and gastric cancer: a hospital-based case-control study. Biomarkers in medicine. 2013; 7: 131-7.

6. Chiang SF, Hung HY, Tang R, Changchien CR, Chen JS, You YT, et al. Can neutrophil-to-lymphocyte ratio predict the survival of colorectal cancer patients who have received curative surgery electively? International journal of colorectal disease. 2012; 27: 1347-57.

7. Teramukai S, Kitano T, Kishida Y, Kawahara M, Kubota K, Komuta K, et al. Pretreatment neutrophil count as an independent prognostic factor in advanced non-small-cell lung cancer: an analysis of Japan Multinational Trial Organisation LC00-03. European journal of cancer (Oxford, England : 1990). 2009; 45: 1950-8.

8. He WZ, Jiang C, Yin CX, Guo GF, Rong RM, Qiu HJ, et al. Prognostic model built on blood-based biomarkers in patients with metastatic colorectal cancer. Asian Pacific journal of cancer prevention : APJCP. 2014; 15: 7327-31. 
9. Yang Q, Liao F, Huang Y, Jiang C, Liu S, He W, et al. Longterm effects of palliative local treatment of incurable metastatic lesions in colorectal cancer patients. Oncotarget. 2016; 7: 21034-45.

10. He W, Yin C, Guo G, Jiang C, Wang F, Qiu H, et al. Initial neutrophil lymphocyte ratio is superior to platelet lymphocyte ratio as an adverse prognostic and predictive factor in metastatic colorectal cancer. Medical oncology (Northwood, London, England). 2013; 30: 439.

11. Saltz LB, Clarke S, Diaz-Rubio E, Scheithauer W, Figer A, Wong R, et al. Bevacizumab in combination with oxaliplatin-based chemotherapy as first-line therapy in metastatic colorectal cancer: a randomized phase III study. Journal of clinical oncology : official journal of the American Society of Clinical Oncology. 2008; 26: 2013-9.

12. Yang Q, Yin C, Liao F, Huang $Y$, He W, Jiang C, et al. Bevacizumab plus chemotherapy as third- or later-line therapy in patients with heavily treated metastatic colorectal cancer. OncoTargets and therapy. 2015; 8: 2407-13.

13. Bennouna J, Sastre J, Arnold D, Osterlund P, Greil R, Van Cutsem E, et al. Continuation of bevacizumab after first progression in metastatic colorectal cancer (ML18147): a randomised phase 3 trial. The Lancet Oncology. 2013; 14: 29-37.

14. Jubb AM, Harris AL. Biomarkers to predict the clinical efficacy of bevacizumab in cancer. The Lancet Oncology. 2010; 11: 1172-83.

15. Lambrechts D, Lenz HJ, de Haas S, Carmeliet P, Scherer SJ. Markers of response for the antiangiogenic agent bevacizumab. Journal of clinical oncology : official journal of the American Society of Clinical Oncology. 2013; 31: 1219-30.

16. Yin C, Jiang C, Liao F, Rong Y, Cai X, Guo G, et al. Initial LDH level can predict the survival benefit from bevacizumab in the first-line setting in Chinese patients with metastatic colorectal cancer. OncoTargets and therapy. 2014; 7: 1415-22.

17. Zhang WHB. A Potential Administration-time Dependent Effect of Bevacizumab in Improving Overall Survival and Increasing Metastasis in Metastatic Colorectal Cancer. Chemotherapy Open Access. 2013; 02.

18. Yin C, Ma G, Rong Y, Kong P, Yang Q, Jiang C, et al. The Efficacy of Bevacizumab in Different Line Chemotherapy for Chinese Patients with Metastatic Colorectal Cancer. Journal of Cancer. 2016; 7: 1901-6.

19. Zhang W, Azuma M, Lurje G, Gordon MA, Yang D, Pohl A, et al. Molecular predictors of combination targeted therapies (cetuximab, bevacizumab) in irinotecan-refractory colorectal cancer (BOND-2 study). Anticancer research. 2010; 30: 4209-17.

20. Duffy MJ, van Dalen A, Haglund C, Hansson L, Klapdor R, Lamerz R, et al. Clinical utility of biochemical markers in colorectal cancer: European Group on Tumour Markers (EGTM) guidelines. European journal of cancer (Oxford, England : 1990). 2003; 39: 718-27.

21. Locker GY, Hamilton S, Harris J, Jessup JM, Kemeny N, Macdonald JS, et al. ASCO 2006 update of recommendations for the use of tumor markers in gastrointestinal cancer. Journal of clinical oncology : official journal of the American Society of Clinical Oncology. 2006; 24: 5313-27.

22. Van Cutsem E, Nordlinger B, Cervantes A. Advanced colorectal cancer: ESMO Clinical Practice Guidelines for treatment. Annals of oncology : official journal of the European Society for Medical Oncology. 2010; 21 Suppl 5: v93-7.

23. Zhang LN, OuYang PY, Xiao WW, Yu X, You KY, Zeng ZF, et al. Elevated CA19-9 as the Most Significant Prognostic Factor in Locally Advanced Rectal Cancer Following Neoadjuvant Chemoradiotherapy. Medicine. 2015; 94: e1793.

24. Filella X, Molina R, Grau JJ, Pique JM, Garcia-Valdecasas JC, Astudillo E, et al. Prognostic value of CA 19.9 levels in colorectal cancer. Annals of surgery. 1992; 216: 55-9.

25. Kouri M, Pyrhonen S, Kuusela P. Elevated CA19-9 as the most significant prognostic factor in advanced colorectal carcinoma. Journal of surgical oncology. 1992; 49: 78-85.

26. Nolen BM, Brand RE, Prosser D, Velikokhatnaya L, Allen PJ, Zeh HJ, et al. Prediagnostic serum biomarkers as early detection tools for pancreatic cancer in a large prospective cohort study. PloS one. 2014; 9: e94928.

27. Tampellini M, Ottone A, Alabiso I, Baratelli C, Forti L, Berruti A, et al. The prognostic role of baseline CEA and CA 19-9 values and their time-dependent variations in advanced colorectal cancer patients submitted to first-line therapy. Tumour biology : the journal of the International Society for Oncodevelopmental Biology and Medicine. 2015; 36: 1519-27.

28. Hanke B, Riedel C, Lampert S, Happich K, Martus P, Parsch H, et al. CEA and CA 19-9 measurement as a monitoring parameter in metastatic colorectal cancer $(C R C)$ under palliative first-line chemotherapy with weekly 24-hour infusion of high-dose 5-fluorouracil (5-FU) and folinic acid (FA). Annals of oncology : official journal of the European Society for Medical Oncology. 2001; 12: $221-6$

29. Zheng $\mathrm{CX}$, Zhan $\mathrm{WH}$, Zhao JZ, Zheng D, Wang DP, He $\mathrm{YL}$, et al. The prognostic value of preoperative serum levels of CEA, CA19-9 and CA72-4 in patients with colorectal cancer. World journal of gastroenterology. 2001; 7: 431-4.

30. Formica V, Massara MC, Portarena I, Fiaschetti V, Grenga I, Del Vecchio Blanco G, et al. Role of CA19.9 in predicting bevacizumab efficacy for metastatic colorectal cancer patients. Cancer biomarkers : section A of Disease markers. 2009; 5: 167-75.

31. Narita Y, Taniguchi H, Komori A, Nitta S, Yamaguchi K, Kondo C, et al CA19-9 level as a prognostic and predictive factor of bevacizumab efficacy in metastatic colorectal cancer patients undergoing oxaliplatin-based chemotherapy. Cancer chemotherapy and pharmacology. 2014; 73: 409-16.

32. Petrioli R, Licchetta A, Roviello G, Pascucci A, Francini E, Bargagli G, et al. CEA and CA19.9 as early predictors of progression in advanced/metastatic colorectal cancer patients receiving oxaliplatin-based chemotherapy and bevacizumab. Cancer investigation. 2012; 30: 65-71.

33. Fiala O, Finek J, Buchler T, Matejka VM, Holubec L, Kulhankova J, et al. The Association of Serum Carcinoembryonic Antigen, Carbohydrate Antigen 19-9, Thymidine Kinase, and Tissue Polypeptide Specific Antigen with Outcomes of Patients with Metastatic Colorectal Cancer Treated with Bevacizumab: a Retrospective Study. Targeted oncology. 2015; 10: 549-55.

34. Kayser G, Kassem A, Sienel W, Schulte-Uentrop L, Mattern D, Aumann K, et al. Lactate-dehydrogenase 5 is overexpressed in non-small cell lung cancer and correlates with the expression of the transketolase-like protein 1 . Diagnostic pathology. 2010; 5: 22

35. Danner BC, Didilis VN, Wiemeyer S, Stojanovic T, Kitz J, Emmert A, et al. Long-term survival is linked to serum LDH and partly to tumour LDH-5 in NSCLC. Anticancer research. 2010; 30: 1347-51.

36. Hong J, Yoon HH, Ahn HK, Sym SJ, Park J, Park PW, et al. Prognostic role of serum lactate dehydrogenase beyond initial diagnosis: a retrospective analysis of patients with diffuse large B cell lymphoma. Acta haematologica. 2013; 130: 305-11.

37. Jiang $\mathrm{C}, \mathrm{Hu} \mathrm{WM}$, Liao FX, Yang $\mathrm{Q}$, Chen $\mathrm{P}$, Rong $\mathrm{YM}$, et al. Elevated preoperative neutrophil-to-lymphocyte ratio is associated with poor prognosis in gastrointestinal stromal tumor patients. OncoTargets and therapy. 2016; 9: 877-83. 